\title{
PERFORMANCE COMPARISON OF CLASSIC AND FUZZY LOGIC CONTROLLERS FOR COMMUNICATION AIRSHIPS
}

\author{
Maryam Falahpour, Hassan Moradi, Hazem H. Refai, Mohammed Atiquzzaman, \\ Electrical and Computer Engineering, University of Oklahoma, Tulsa, OK. \\ Robert Rubin, Peter G. LoPresti, Electrical Engineering Department, University of Tulsa, Tulsa, OK.
}

\begin{abstract}
Communication is vital in order to coordinate rescue and clean up efforts should an event eliminate the most common infrastructure, such a cell tower and phone lines, in a region. One of the most effective ways to set up an alternative communication system without relying on traditional methods would be to attach a temporary system to airships floating above the region. However, an inherent problem with airships is keeping them in the desired area with the correct orientation. This paper compares two different airship control methods, namely fuzzy logic and classic controllers, to navigate the balloon while hovering over a region in order to reestablish communication. A dynamical model of the airship in the presence of air friction is shown, and simulations are done using MATLAB Simulator.
\end{abstract}

\section{Introduction}

When a natural catastrophe, e.g. hurricane or earthquake, affects a region, cellular communication may cease and towers can be damaged, causing the loss of vital communication at a time when it is most important. Hence, it is essential to rapidly deploy a replacement system independent of infrastructure to facilitate communication among rescuers, enabling aid to distressed people and expediting recovery efforts. This research considers an airborne communication network that can be rapidly deployed to establish a replacement method of communication. There is immense potential in utilizing robotic airships as low speed, low altitude aerial vehicles in exploration and monitoring tasks. Blimps have several useful properties, including ease in hovering, reduced fuel consumption and less noise. Unmanned blimp systems have recently been used for advertisements and atmospheric analysis. Unmanned aerial vehicles (UAVs) play an important role in military reconnaissance and surveillance missions, and agencies such as NASA are employing aerial vehicles as platforms for environmental and climate research.

Airships provide some of the most effective platforms for research because they are able to hover over an area and make observations for an extended period of time without disturbing environment. Airships generate little vibration thereby reducing sensor noise and hardware malfunction. Also, they can take off and land vertically without runways. Airships can also reach anywhere so that remote or difficult to access regions can be monitored. They have a large payload capacity and have low operation cost [1].

In light of the aforementioned benefits, controllable balloon studies are of great importance for rapidly deployed airborne communication systems. In this paper we compare the performance of two methods applicable for controlling the position and direction of communication balloons. The first is a feedback linearization controller, which is categorized as one of the classic methods in control. The second is a fuzzy logic controller, commonly used when the exact conditions of the model are unknown.

\section{Related Works}

Previous research with regard to balloon control exists in the literature. Physical principles of airship operations using nonlinear dynamics to control several flight phases from takeoff to landing are described in [2, 3]. Also, Zhang and Ostrowski [4] use a vision-guided blimp combined with a classical PID controller. Furthermore [5] discusses imagebased tracking control for an indoor blimp, while Wyeth and Barrons [6] present a low level reactive controller. A learning algorithm has been presented in [7], which does not assume prior knowledge about dynamics or pre-defined controller. Instead, it develops the control policy online and does not 
require a-priori information about the payload, temperature or air pressure.

Time delay and disturbance are two challenges when controlling a blimp. State predictive control can be applied to an autonomous blimp in the presence of time delay and disturbance [8]. In that work, model predictive control (MPC) has been applied to an autonomous blimp, and an indoor flight experiment has been applied to an autonomous blimp to evaluate its effectiveness. With focus on position control, a constrained MPC for an autonomous blimp has been discussed in [9]. Image processing and vision-based techniques are common for tracking mobile blimps [10]. Image-based tracking control of an aerial blimp is proposed for surveillance systems. In this scenario, the controller is designed by back-stepping techniques for under-actuated systems.

In this paper, the classic and fuzzy controllers are simulated and compared to determine which controller best fit to maintain position and orientation for balloon-to-balloon optical communication. This is a work in progress to develop a fast deployable wireless optical/RF hybrid communication system using airships.

\section{System Model and Dynamics}

Once communication in a defined area is disrupted, a group of balloons will be launched to restore communication. The balloons must maintain a specified distance from each other. Because the balloons are equipped with optical transceiversenabling higher data rate than $\mathrm{RF}$-orientation is vital for sustained communication between balloons. The controllers investigated in this paper simulate a single balloon reaching a certain position and maintaining its orientation in spite of adverse wind condition.

The balloon will be equipped with a GPS for position and an electronic compass for direction. Also, the balloon is equipped with a 3-axis accelerometer to measure small changes in position. The propulsion system consists of three propellers assembled at either side of the center of gravity and at the tail. Propellers use three brushless motors and a motor speed controller.

Figure 1 shows a model of the balloon used in this paper. As shown, the balloon used in the unmanned airship system has three propellers- two assembled at either side of the center of gravity of the balloon and one at the tail. We assume the forces of these propellers operate only the horizontal direction. In Figure 1, $\mathbf{x}$ and $\mathbf{y}$ denote the position of the center of gravity of the balloon, and $\theta$ denotes the angle from $\mathbf{x}$ axis to the direction of movement of the balloon [11]. For simplicity we also assume that the balloon is a rigid body and has no rolling, no pitching and no vertical movement.

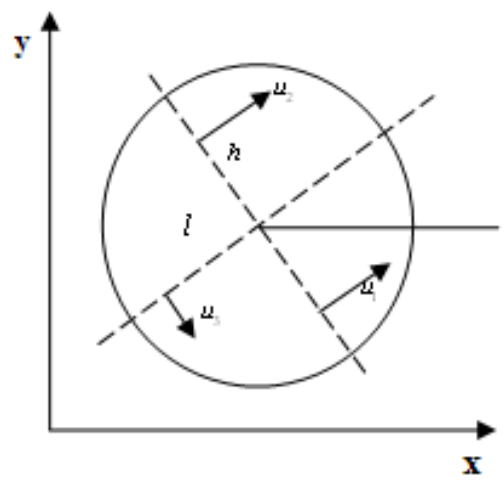

Figure 1. A Model for the Balloon

The parameters shown in Figure 1 are defined as follows:

- $u_{1}$ and $u_{2}$ and $u_{3}:[\mathrm{N}]$ thrusts of the propellers

- $h$ and $l:[\mathrm{m}]$ the distance from the center of gravity of the balloon to either side of the propellers and from the center of gravity to the back propeller.

The dynamical equations are derived as follows:

$m \ddot{x}=u_{1} \cos \theta+u_{2} \cos \theta+u_{3} \sin \theta-f_{f x}$

$m \ddot{y}=u_{1} \sin \theta+u_{2} \sin \theta-u_{3} \cos \theta-f_{f y}$

$J \ddot{\theta}=h\left(u_{1}-u_{2}\right)+l u_{3}$

The parameters in the equation (1) are defined as follows:

- $m$ : mass of the balloon.

- $J$ : [kg.m2] the moment of inertia around the center of gravity of the balloon.

- $f_{f x}$ and $f_{f y}$ : the force of air friction for the $\mathbf{x}$ and $\mathbf{y}$ axis respectively. A simple air friction is used to using the following model:

$$
f_{f}=-\frac{1}{2} C \rho A v^{2}
$$


where $\rho$ is the density of the fluid. (Note that for the earth's atmosphere, the density can be found using the barometric formula: $1.293 \mathrm{~kg} / \mathrm{m}^{3}$ at $0{ }^{\circ} \mathrm{C}$ and 1 atmosphere). Also, $v$ is the speed of the object relative to the fluid; $A$ is the reference area; and $C$ as a dimensionless parameter is the drag coefficient. The balloon position (i.e. $\mathbf{x}$ and $\mathbf{y}$ ) will be provided by GPS receiver, while the direction $\theta$ will be given by the onboard electronic compass.

The performance is evaluated to accomplish two objectives: 1.) the ability to navigate the balloon to pre-specified destination 2.) the ability to maintain balloon orientation in presence of wind disturbance.

\section{Feedback Linearization Controller}

\section{A. The Theory}

In recent years, feedback linearization to nonlinear control design has attracted the attention of researchers. The goal is to algebraically transform nonlinear systems dynamics into full or partial linear systems so that linear control techniques can be applied. This paper does not discuss the concept of this method, as valuable details can easily be found in related works (e.g. [12]). This paper deploys a feedback linearization controller for the balloon system as a classic controller. Corresponding results are be presented.

The method may be applied to nonlinear systems of the form:

$$
\begin{aligned}
& \dot{x}=f(x)+g(x) u \\
& y=h(x)
\end{aligned}
$$

where $x \in R^{n}$ is the state vector, $u \in R^{p}$ is the vector of inputs, and $y \in R^{m}$ is the vector of outputs. By applying the Lie Derivative [12] to the output, we get:

$\dot{y}=\frac{\partial h}{\partial x}(f(x)+g(x) u)=L_{f} h(x)+L_{g} h(x) u$

$L_{g}$ and $L_{f}$ are Lie derivatives for $g$ and $f$ functions. If $L_{g} h(x) u \neq 0$ then

$$
\ddot{y}=\frac{\partial\left(L_{f} h\right)}{\partial x}(f(x)+g(x) u)=L_{f}^{2} h(x)+L_{g} L_{f} h(x) u
$$

But if $L_{g} L_{f} h(x) u \neq 0$, we must continue to apply the Lie Derivative until $u$ appears in the equation:

$y^{\rho}=L_{f}^{\rho} h(x)+L_{g} L_{f}^{\rho-1} h(x) u$

where $1<\rho<n$. Then the control effort signal will be given by

$u=\frac{1}{L_{g} L_{f}^{\rho-1} h(x)}\left[-L_{f}^{\rho} h(x)+v\right]$

where $\mathrm{v}$ is a linear PD controller. In our system the final control effort is defined as follows:

$$
\begin{aligned}
& G=\left[\begin{array}{ccc}
\frac{\cos \theta}{m} & \frac{\cos \theta}{m} & \frac{\sin \theta}{m} \\
\frac{\sin \theta}{m} & \frac{\sin \theta}{m} & -\frac{\cos \theta}{m} \\
\frac{h}{J} & -\frac{h}{J} & \frac{l}{J}
\end{array}\right] \\
& \left.u=G^{-1}\left[\begin{array}{c}
f_{f x} \\
f_{f y} \\
0
\end{array}\right]+K p \cdot e+K d \cdot \dot{e}\right]
\end{aligned}
$$

where $\mathrm{Kp}, \mathrm{Kd}$, e and ${ }^{e}$ are defined as follow:

$$
K p=\left[\begin{array}{lll}
1 & 0 & 0 \\
0 & 1 & 0 \\
0 & 0 & 5
\end{array}\right], K d=\left[\begin{array}{lll}
1 & 0 & 0 \\
0 & 1 & 0 \\
0 & 0 & 1
\end{array}\right], e=\left[\begin{array}{l}
x_{d}-x \\
y_{d}-y \\
\theta_{d}-\theta
\end{array}\right], e=\left[\begin{array}{l}
\dot{x}_{d}-\dot{x} \\
\dot{y}_{d}-\dot{y} \\
\dot{\theta}_{d}-\dot{\theta}
\end{array}\right]
$$

In this equations, $x_{d}, y_{d}$ and $\theta_{d}$ are the desired position and angle of the balloon and $x, y$ and $\theta$ are the current position and angle. The matrix $G$ is nonsingular for any value of $x, y$ and $\theta$. By calculating the determinant of the matrix $G$ in (8) we have [2]:

$$
\operatorname{det}\{G\}=-\frac{2 h}{J m^{2}}
$$

Since $\mathrm{h}$ is not equal to zero for any value of $x, y$ and $\theta, \operatorname{det}\{\mathrm{G}\}$ cannot equal zero. Thus, the matrix $\mathrm{G}$ is nonsingular for any value of $x, y$ and $\theta$.

\section{B. Simulation}

A control system based on feedback linearization technique has been developed and applied on the balloon system. The control scheme is shown in 
Figure 2. In this figure, $\vec{y}_{d}$ is a matrix of desired signals or our reference point and $\vec{y}_{p}$ is the current position of balloon. The vector $\vec{u}$ is the output of the controller. Disturbance on the balloon is due to only to wind. Its effect on the balloon is simulated as force with changing magnitude and direction. Simulation results for this controller are presented in Figures 3-6: one showing a block diagram, one the trajectory of the balloon, while others illustrates the angle and control effort signals applied on the propellers $u_{1}, u_{2}$, and $u_{3}$.

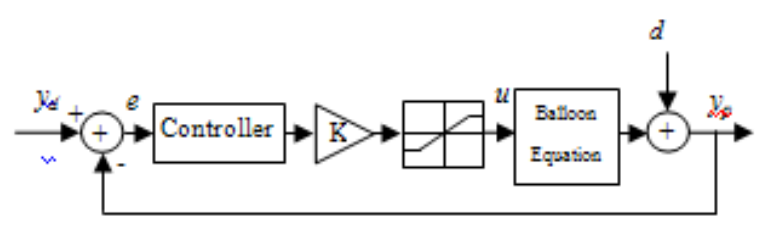

Figure 2. Control Diagram

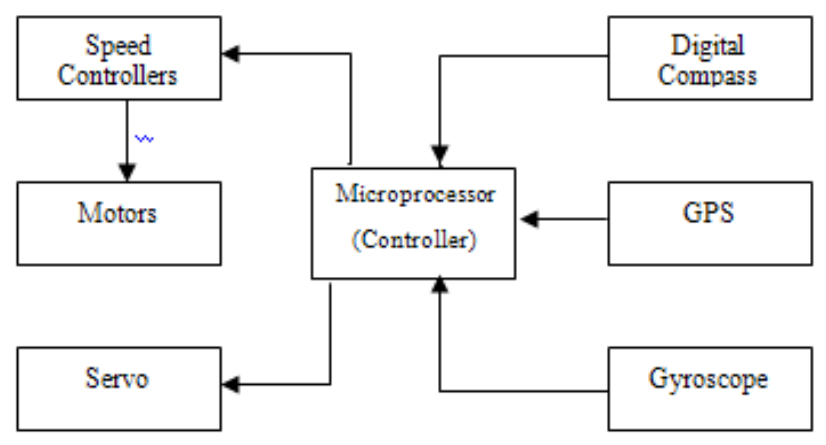

Figure 3. Block Diagram of Airship's Sensors/Actuators

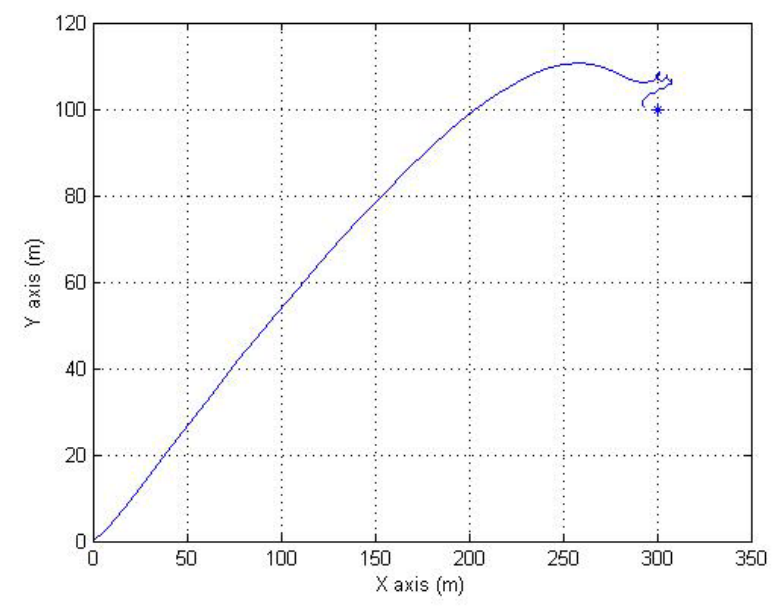

Figure 4. X-Y Trajectory
Note that the effect of wind simulation took into consideration the fact that the wind's direction and force are not constant. It can be inferred from Figures 3 and 4 that the balloon is able to achieve the desired point after approximately 10 minutes. At this interval, a small amount of power still exists a on the propellers. Changes on the control effort signals are within acceptable range and converge.

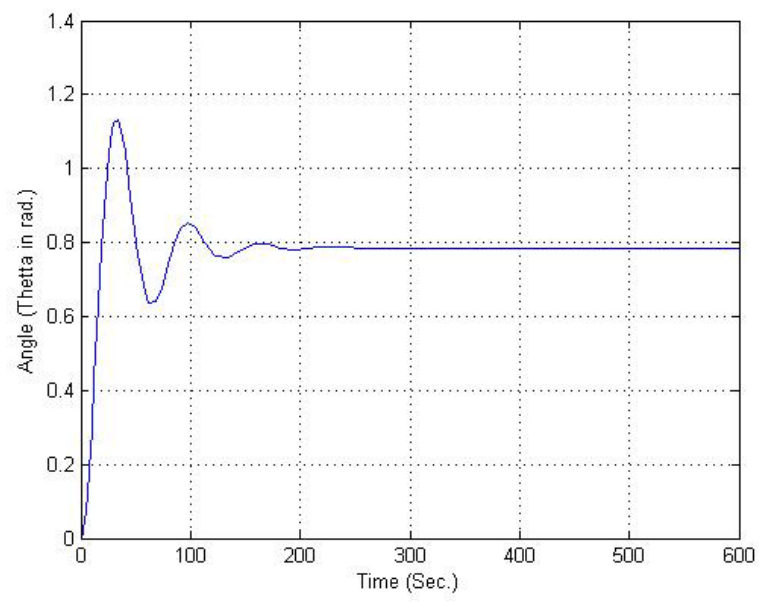

Figure 5. Angle ( $\theta$ )
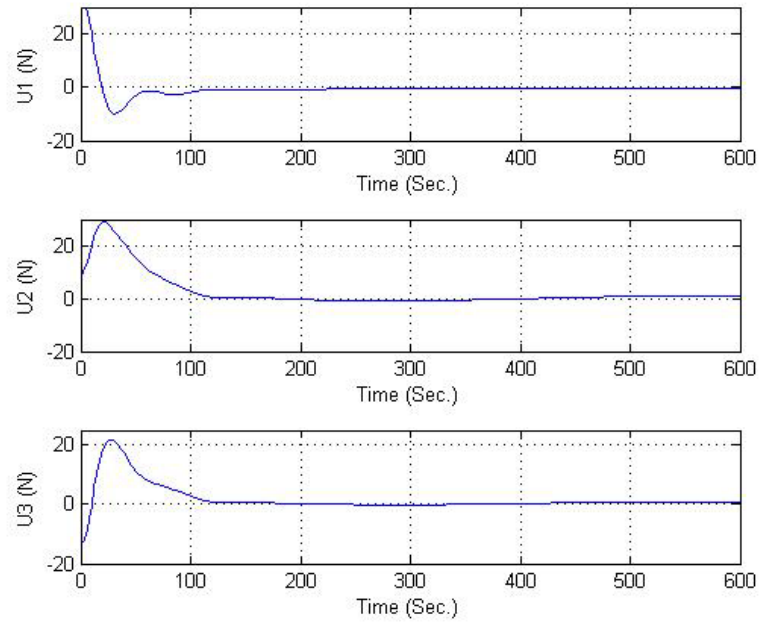

Figure 6. Control Efforts $\left(u_{1}, u_{2}, u_{3}\right)$

\section{Fuzzy Controller Description}

\section{A. The Principles}

Balloon Model has unknown parameters, thus require a sophisticated design. A fuzzy logic controller is a good choice for systems with 
parametric perturbation. However, the system requires substantial knowledge and experience from a skilled operator. The controller must have the capability to immediately correct any errors detected in the system. (Interested readers may find useful information about fuzzy logic control in [13, 14].)

In the control system researched in this paper, the control procedure is divided to two parts or subcontrollers, such that the controller may use one in a given time. The first is for navigation and is used from the place of origin until the desired point is achieved. The corresponding sub-controller attempts to direct the balloon to the destination at the desired point. The second is designed for orientation purposes, which denotes the direction of the balloon after reaching the desired point. Since the balloon should maintain a directional wireless communication system, this latter task is an extremely important factor in the design.

Data received from sensors attached to the balloon goes to the controller, where it converted into fuzzy variables. This function is called the fuzzification process. The fuzzified data then goes through a set of if-then rules in an inference engine, which results in fuzzy outputs that are converted back to a crisp value through a process called defuzification by the weighted average method [15].

Defining the membership functions for input/output variables is the first step for fuzzification. A membership function is a graphical representation of the magnitude of participation for each input. It associates a weighting with each of the inputs that are processed, defines functional overlap between inputs, and ultimately determines an output response. Some control rules are required to use the input membership values as weighting factors to determine their influence on the fuzzy output sets of the final output conclusion. Once the functions are inferred, scaled, and combined, they are defuzzified into a crisp output, which drives the system. Different membership functions may be associated with each input and output response [14].

In this paper, three and five membership functions with triangular shapes are considered for each input and output variable, respectively. They are illustrated in Figures 7 and 8. Different states are assigned to controlled variables to represent the state of each variable. The values of states might be Small (S), Normal (N), Big (B) and for the control efforts might be Negative Big (NB), Negative Small (NS), Normal (N), Positive Small (PS) and Positive Big (PB). The instructions which determine the relation between controlled variables and control efforts are denoted above as control rules.

The input errors for navigation system are $x_{d}-x, y_{d}-y, \theta_{d}^{\prime}-\theta$ and $\dot{\theta}_{d}-\dot{\theta}$; which $\theta_{d}^{\prime}$ is the angle between the current position of the balloon and the desired point, and for the orientation system the inputs are $\theta_{d}-\theta$ and $\dot{\theta}_{d}-\dot{\theta}$. Rules are generated based on the number of inputs for navigation controller $3^{4}=81$ rules and for the orientation controller $3^{2}=9$ rules. The rules defined for the orientation control are listed in Table 1.

\section{Table 1. Control Rules for Orientation}

\begin{tabular}{|l|l|l|l|l|}
\hline Rule \# & $\theta_{d}-\theta$ & $\dot{\theta}_{d}-\dot{\theta}$ & $u_{1}$ & $u_{2}$ \\
\hline Rule1 & $\mathrm{S}$ & $\mathrm{S}$ & $\mathrm{NS}$ & $\mathrm{PB}$ \\
\hline Rule2 & $\mathrm{S}$ & $\mathrm{N}$ & $\mathrm{S}$ & $\mathrm{B}$ \\
\hline Rule3 & $\mathrm{S}$ & $\mathrm{B}$ & $\mathrm{N}$ & $\mathrm{N}$ \\
\hline Rule4 & $\mathrm{N}$ & $\mathrm{S}$ & $\mathrm{S}$ & $\mathrm{B}$ \\
\hline Rule5 & $\mathrm{N}$ & $\mathrm{N}$ & $\mathrm{N}$ & $\mathrm{N}$ \\
\hline Rule6 & $\mathrm{N}$ & $\mathrm{B}$ & $\mathrm{B}$ & $\mathrm{S}$ \\
\hline Rule7 & $\mathrm{B}$ & $\mathrm{S}$ & $\mathrm{N}$ & $\mathrm{N}$ \\
\hline Rule8 & $\mathrm{B}$ & $\mathrm{N}$ & $\mathrm{B}$ & $\mathrm{S}$ \\
\hline Rule9 & $\mathrm{B}$ & $\mathrm{B}$ & $\mathrm{PB}$ & $\mathrm{NS}$ \\
\hline
\end{tabular}

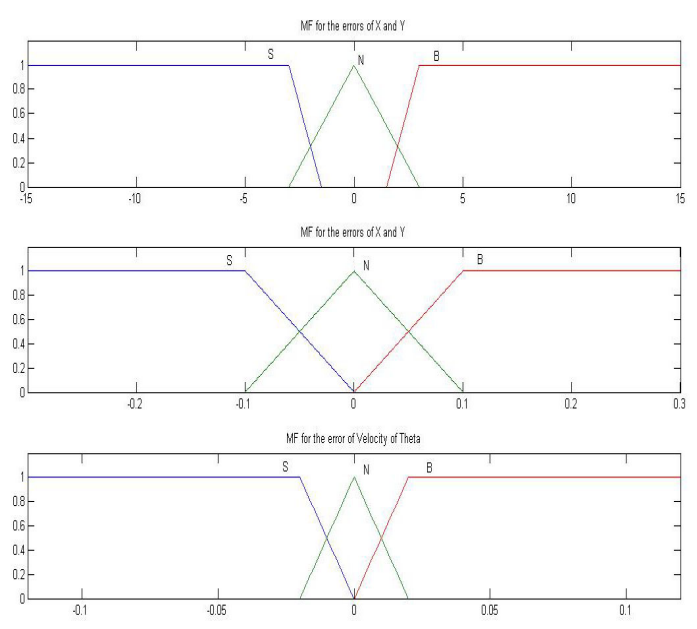

Figure 7. Membership Function of Input Variables 


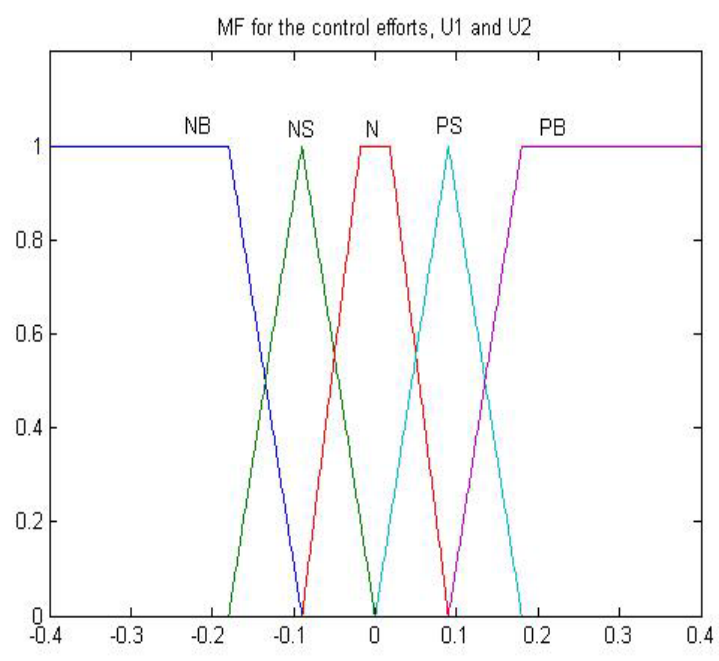

Figure 8. Membership Function of Output Variables

Finally, the control efforts can be formulated by:

$$
u_{i}=\frac{\sum_{j=1}^{n} x_{j} \mu_{j}}{\sum_{j=1}^{n} \mu_{j}} ; i=1,2,3
$$

By applying control effort signals in (8) to the balloon, the simulation results for this controller can be shown as in Figures 9, 10 and 11. The simulation has been done in the presence of wind. The wind's direction and magnitude is not constant. As shown in Figure 9, balloon is able to achieve the desired point. The oscillation of the control efforts in Figure 11 at the beginning are less than the classic controller in previous section. It can be concluded that this controller can work best if there is any limitation in the real control efforts; however, its total performance is not as good as the classic controller in the presence of the wind disturbance.

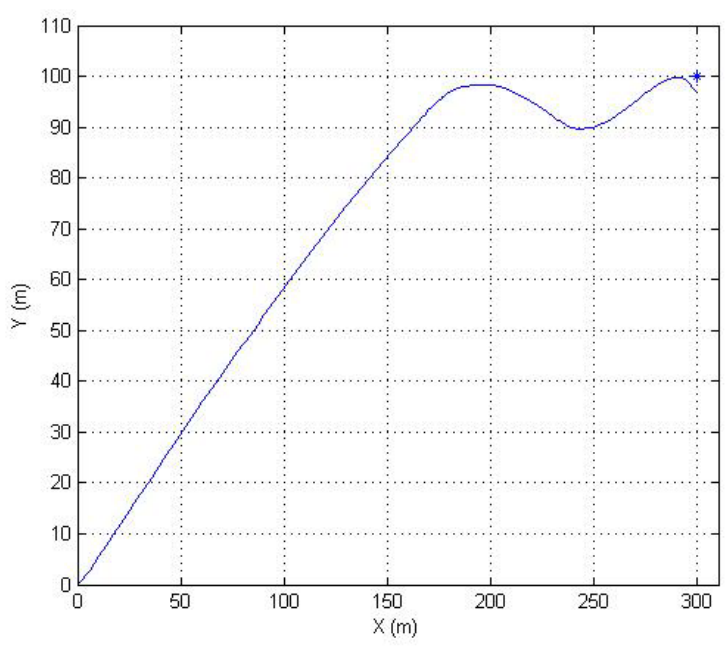

Figure 9. XY Trajectory

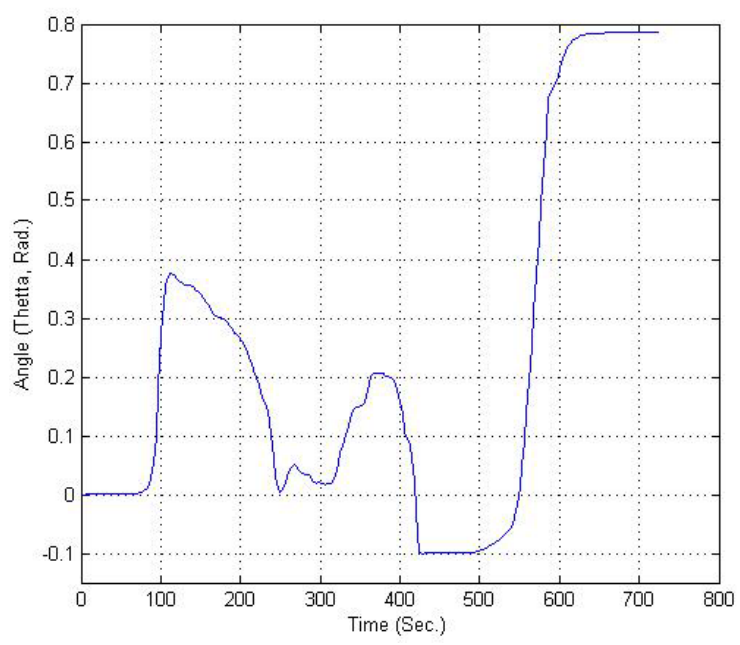

Figure 10. Angle ( $\theta)$
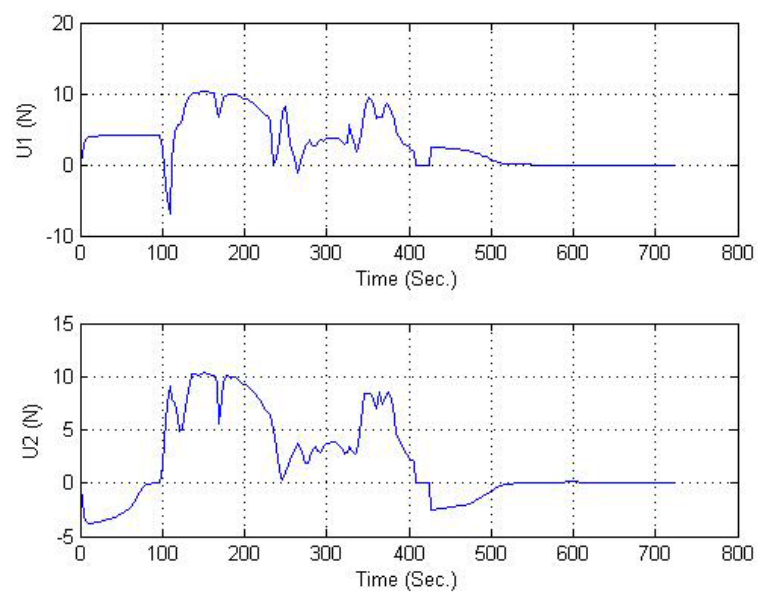

Figure 11. Control Efforts $\left(u_{1}, u_{2}\right)$ 


\section{Result Discussion}

The results shown in the balloon trajectory, controller efforts, and response timings per tested controller scheme are discussed in this section. When the classical controller with feedback linearization is used, faster response time is achieved comparing to when a fuzzy controller is used. A fast response makes the system faster to reach its steady state in the presence of disturbance, as shown in Figures 4-6. Using classical controller, the balloon flight time to destination located at 316 meters $(300 \mathrm{~m}, 100 \mathrm{~m})$ away is simulated to be 300 seconds. It is observed that the balloon is not able to arrive at the exact destination due to wind disturbance. The position error is limited to between 3 and 4 meters. No position error is observed with no wind disturbance. The controller is able to place the balloon on the destination. On the other hand, the fuzzy controller arrives at destination in approximately 420 seconds.

The models used in the simulation are selected to be simple: $2^{\text {nd }}$ order balloon dynamic model, velocity-squared friction model, and non-constant force for wind disturbances. Hence, the classical controller out performed the fuzzy controller. However, the uncertainties in a practical balloon navigation and deployment may make the classical controller under perform, while fuzzy controller may perform better with the uncertainties.

Both controllers use the same hardware for collecting attitude data and navigation the balloon. In terms of the MATLAB simulation time, the fuzzy controller requires more time to compute control efforts, while the classic controller does the computation much faster.

\section{Conclusion}

This paper considers a controllable airborne platform to employ a wireless communication network that can be rapidly deployed to establish communication service in a distressed area. The performance of two alternative controllers (classic and fuzzy) for balloon navigation is analyzed and simulated using MATLAB. The simulation results indicate that the classical controller out performs the fuzzy controller in achieving destination with approximately 120 seconds less.

\section{References}

[1] A. Elfes, S. S. Bueno, M. Bergerman, J. J. G. Ramos, May 1998, A Semi-Autonomous Robotic Airship for Environmental monitoring Missions, Proceedings of the IEEE International Conference on Robotics \& Automation, pp. 3449-3455, Belgium.

[2] S. Varella Gomes and J. Ramos, 1998, Airship dynamic modeling for autonomous operation, In Proc. of the IEEE Int. Conf. on Robotics \& Automation (ICRA), pp. 3462-3467.

[3] E. Hygounenc, I-K. Jung, P. Soueres, and S. Lacroix, 2004, The autonomous blimp project at LAAS/CNRS: Achievements in flight control and terrain mapping, In International Journal of Robotics Research. , pp. 473-511.

[4] H. Zhang and J. Ostrowski, 1999, Visual servoing with dynamics: Control of an unmanned blimp, In Proc. of the IEEE Int. Conf. on Robotics \& Automation (ICRA), pp. 618-623.

[5] T. Fukao, K. Fujitani, T. Kanade, 2003, Imagebased Tracking Control of a Blimp, the 42nd IEEE Conference on Decision and Control, pp. 5414-5419.

[6] G. Wyeth and I. Barron, , 1997, An autonomous blimp, In Proc. of the IEEE Int. Conf. on Field and Service Robotics (FSR), pp. 464-470.

[7] A. Rottmann, C. Plagemann, P. Hilgers, 2007, W. Burgard, Autonomous Blimp Control using Modelfree Reinforcement Learning in a Continuous State and Action Space, IEEE/RSJ International Conference on Intelligent Robots and Systems, pp. 1895-1900.

[8] H. Fukushima, K. Kon, Y. Hada, F. Matsuno, K. Kawabata, H. Asama, 2007, State-Predictive Control of an Autonomous Blimp in the Presence of Time Delay and Disturbance, 16th IEEE International Conference on Control Applications Part of IEEE Multi-conference on Systems and Control, pp. 188193.

[9] H. Fukushimal, K. Kon, F. Matsunol, Y. Hada, K. Kawabata, H. Asama, 2006, Constrained Model Predictive Control: Applications to Multi-Vehicle Formation and an Autonomous Blimp, SICE-ICASE International Joint Conference, pp. 4515-4520.

[10] L. D. S. COELHO1, M. F. M. CAMPOS1, V. KUMAR, 1998, Computer Vision-Based Navigation for Autonomous Blimps, International Symposium 
on Computer Graphics, Image Processing, and Vision (SIBGRAPI), pp. 287 - 294.

[11] Yasunori Kawai, Satoshi Kitagawa, Shintaro Izoel and Masayuki Fujita, August 4-6 2003, An Unmanned Planar Blimp on Visual Feedback Control : Experimental Results. SICE Annual Conference in Fukui, Fukui University, Japan. pp. 2217-2222

[12] Hassan K. Khalil, 2001, Nonlinear Systems, Third edition, prentice Hall.

[13] D. Driankov, H. Hellendoorn, M.. Reinfrank, L. Ljung, R. Palm, 1996, An Introduction to Fuzzy Control, Springer, 2nd edition.

[14] S.D. Kaehler, Fuzzy Logic-An Introduction, available

at: http://www.seattlerobotics.org/encoder/mar98/fuz/ flindex.html

[15] Guo Jian-guo, Zhou Jun, Dec. 2008, Altitude Control System of Autonomous Airship Based on Fuzzy Logic, 2nd International Symposium on
Systems and Control in Aerospace and Astronautics, 2008. ISSCAA 2008. pp:1-5.

\section{Acknowledgement}

This work is funded by NSF grant number NSFECCS 0725801.

\section{Email Addresses}

Maryam Falahpour: maryam@ou.edu

Hassan Moradi: $\underline{\text { hmoradi@ou.edu }}$

Hazem H. Refai: $\underline{\text { hazem@ou.edu }}$

Mohammed Atiquzzaman: atiq@ou.edu

Peter G. LoPresti: pgl@ohm.ee.utulsa.edu

28th Digital Avionics Systems Conference

October 25-29, 2009 